\title{
Quelques remarques sur l'enseignement des langues étrangères (à l'exception du français) en Flandre au XIXe siècle
}

\section{Michel Berré}

\section{(2) OpenEdition Journals}

Édition électronique

URL : https://journals.openedition.org/dhfles/2061

DOI : $10.4000 /$ dhfles.2061

ISSN : 2221-4038

Éditeur

Société Internationale pour l'Histoire du Français Langue Étrangère ou Seconde

Édition imprimée

Date de publication : 1 juin 2001

Pagination : 105-119

ISSN : 0992-7654

\section{Référence électronique}

Michel Berré, «Quelques remarques sur l'enseignement des langues étrangères (à l'exception du français) en Flandre au XIXe siècle », Documents pour l'histoire du français langue étrangère ou seconde [En ligne], 26 | 2001, mis en ligne le 14 juillet 2012, consulté le 27 mai 2021. URL : http:// journals.openedition.org/dhfles/2061; DOI : https://doi.org/10.4000/dhfles.2061

Ce document a été généré automatiquement le 27 mai 2021.

(c) SIHFLES 


\title{
Quelques remarques sur l'enseignement des langues étrangères (à l'exception du français) en Flandre au XIXe siècle
}

\author{
Michel Berré
}

\section{Préliminaire}

1 L'histoire de l'enseignement des langues en Belgique ne peut être même esquissée sans parallèlement évoquer la "question linguistique belge ", les conflits et les règlements successifs qu'a engendrés la présence sur le territoire de l'actuelle Belgique de plusieurs langues, à savoir, dans l'ordre de leur diffusion, le néerlandais, le français et l'allemand. À proprement parler, seul l'anglais et les langues mortes (le grec et le latin, ce dernier étant encore enseigné dans certaines institutions comme une langue vivante pendant une partie du XIXe siècle) mériteraient le qualificatif de langues étrangères (désormais L2) ${ }^{1}$. Ainsi F. Laurent voyait en 1881 dans l'expression "seconde langue » appliquée à la langue française en Flandre et au flamand en Wallonie un « défaut de classification $»^{2}$. Selon le professeur d'université gantois, il ne fallait faire aucune différence entre les deux langues principales parlées en Belgique. D'autre part, il ne faudrait pas se méprendre sur l'homogénéité des " communautés » linguistiques néerlandophone, francophone et germanophone. À l'intérieur de chacune d'elles existait une grande variété dialectale qui faisait obstacle à la diffusion d'une langue standard en particulier dans les provinces flamandes, difficulté renforcée par la présence d'une population francisée, certes minoritaire (environ 3\%), mais dominante dans le système politique à base censitaire de l'époque. Sans trop d'anachronisme, on pourrait parler pour le milieu du XIXe siècle d'une société "plurilingue », mais d'un plurilinguisme hérité de l'ancien régime où la condition sociale déterminait l'usage des langues ${ }^{3}$. 


\section{Introduction : à propos d'une histoire... sans histoire}

2 La lacune constatée par Chervel (1998:15) à propos des disciplines scolaires dans l'historiographie de l'enseignement en France vaut tout autant pour la Belgique et la Flandre. En ce qui concerne l'enseignement des L2, il faut se contenter de l'ouvrage de Maréchal (1972), ouvrage sans grande valeur théorique. Quant à l'enseignement moyen - niveau essentiellement concerné par la question des «L2 " - l'on se trouve en Belgique, quand il s'agit de le baliser, « sur un terrain presque vierge » (Tyssens 1998 : 223). Dans un tel contexte, la présente contribution se limitera à présenter quelques faits et quelques dates d'une histoire encore à écrire, en focalisant son attention sur le processus d'intégration des L2 dans l'enseignement moyen (essentiellement du premier degré) de l'État.

L'on s'étonnera d'autant plus de l'absence d'étude didactique portant sur l'histoire de l'enseignement des langues en Flandre que l'école y a toujours «baigné » (dans des proportions sans doute plus importantes qu'ailleurs) dans un certain plurilinguisme : bilinguisme entre le patois des élèves et une $\mathrm{Ll}$ en voie de standardisation à l'école élémentaire (d'où le latin n'était pas exclu : éléments de la lecture, prières, etc.) ; bilinguisme flamand français dans toute une série d'écoles « moyennes " (pensionnats, écoles élémentaires à programme développé, etc.) ; bilinguisme flamand latin dans les écoles dites latines (futurs collèges et athénées) ; trilinguisme flamand latin français dans certains collèges plus huppés comme celui des Jésuites à Anvers (fin XVIIe siècle), celui de la Sainte Trinité à Louvain (milieu du XVIIIe siècle) ou encore les collègespensionnats royaux d'après 1777 (cf. infra). D'après Berré (2001), l'histoire de l'enseignement au XIXe siècle peut être interprétée comme le passage d'une école où plusieurs langues avaient droit de cité à une école essentiellement monolingue. Ainsi dans le Dictionnaire de pédagogie de Buisson (fin du XIXe siècle), l'école primaire monolingue apparaît autant comme une évidence que comme une nécessité pédagogique. Ce monolinguisme est bien sûr à mettre en relation avec la constitution des états-nations au XIXe siècle et la construction d'identités nationales fondées sur une communauté de langue et de culture. Il n'interdit pas (au contraire, serait-on tenté de dire), l'enseignement d'autres langues, mais uniquement à l'école secondaire à un moment où la L2 ne peut plus nuire à la $\mathrm{Ll}$ et à ce qu'on appelait le développement intellectuel et moral de l'enfant. Dans ce cadre, la méthode préconisée dans le dernier quart du XIXe siècle est la méthode dite directe qui avait pour principe et effet d'étanchéiser les diverses langues enseignées (notamment par la suppression de la traduction) ; de plus, les L2 étaient enseignées chacune par un professeur différent, selon un horaire spécifique, etc.

4 En Flandre, la situation était cependant quelque peu différente : l'évolution générale vers le monolinguisme était venue buter sur la « nécessité » d'apprendre le français et sur la présence de cette langue à tous les niveaux d'enseignement. Cette présence du français n'empêchait pas les étudiants flamands d'aborder, selon les programmes de l'enseignement moyen de 1850, quatre langues étrangères, outre leur Ll : le latin, le grec, l'allemand et l'anglais. Six langues au total, quel lycéen d'aujourd'hui en étudie davantage? 


\section{De l'Ancien régime à 1850}

\subsection{Avant 1830}

5 La première intervention directe de l'État dans les contenus d'enseignement des collèges date de 1777. Suite à la suppression de la Compagnie de Jésus (1773), le gouvernement autrichien décide de créer une série de collèges et d'imposer à tous les établissements d'enseignement moyen un programme unique (Plan provisionnel d'études 1777) et des manuels scolaires rédigés sous les auspices de la Commission royale des études (1776-1785). Si l'on excepte l'entrée du français dans les cinq collèges-pensionnats les plus importants de Flandre (y compris ceux de Bruxelles et de Ruremonde actuellement aux Pays-Bas), les L2 sont restées en dehors de cette réforme qui visait sur le plan pédagogique à rénover l'étude du latin et à promouvoir l'enseignement des « LI » (flamand français ou allemand selon les régions) et, accessoirement, celui du grec. Sous les régimes français (1794-1815) et hollandais (1815-1830), la législation scolaire en vigueur fut chaque fois celle du pays auquel les anciens Pays-Bas autrichiens furent successivement attachés. Bien que la faculté d'organiser des cours de L2 existât dans les Écoles centrales (1795-1802), elle ne fut pas utilisée (Maréchal 1972 : 45). Le remplacement de ces écoles par des lycées (1802) et la mise sur pied de l'Université impériale (1808-1815) supprimèrent d'ailleurs cette possibilité.

6 Selon Maréchal (op. cit. 62), le régime hollandais fut « le point de départ de l'étude officielle et systématique des langues vivantes étrangères ". L'auteur a ici principalement en vue l'étude obligatoire du néerlandais dans les provinces wallonnes mais il existait aussi dans les provinces méridionales des cours facultatifs de L2 dans les écoles primaires supérieures. Tout enseignant qui souhaitait enseigner une L2 devait obtenir devant une commission un brevet (Sluys 1912 : 265). Pour ce qui est de renseignement moyen de type long, le modèle éducatif est resté celui de l'Ancien régime, sans L2 (Grootaers $1998: 269-270)^{4}$. La période hollandaise est aussi marquée par l'expérience de l'enseignement universel du lecteur de français, Joseph Jacotot, à l'Université de Louvain (1816-1830), expérience dont l'auteur rendit compte - pour ce qui est de l'enseignement des langues - dans deux ouvrages $(1823,1824)$. Ces deux textes suscitèrent une vive polémique et une opposition à Jacotot dans les milieux cultivés francophones de la capitale pour des motifs plus idéologiques que pédagogiques ${ }^{5}$. Sur ce dernier plan, l'influence de Jacotot sur la didactique des LI et des L2 en Belgique a été, à notre avis, sous-estimée.

7 Ce désintérêt des pouvoirs publics pour les L2 ne signifie pas qu'elles n'étaient pas enseignées : elles figuraient au programme de nombreuses institutions privées. Certains pensionnats étaient d'ailleurs en quelque sorte spécialisés dans l'enseignement des L2 comme l'Institut Gaggia à Bruxelles (1829-1845) ${ }^{6}$ organisé en différentes « maisons » (française, hollandaise, anglaise, allemande,...) au sein desquels les matières étaient enseignées aux élèves en différentes langues. Selon Maréchal (op. cit. 34), les L2 pouvaient également être enseignées, à titre facultatif, en dehors des heures de cours et moyennant une rétribution supplémentaire des parents, dans certains collèges. Cet enseignement répondait souvent à une demande locale, d'où le succès de l'anglais en Flandre occidentale ou de l'allemand dans la région liégeoise. 


\subsection{Après 1830}

8 Pour des raisons politiques, la première loi organisant un enseignement moyen de l'État ne sera votée qu'en 1850. Durant les vingt premières années d'existence de la Belgique, l'enseignement moyen vécut sous le régime de la liberté, ce qui aboutit en Flandre à une influence croissante de l'Église ${ }^{7}$ et à une francisation assez poussée de nombreux établissements scolaires. Cette liberté permit aussi à la classe bourgeoise d'intervenir de manière plus directe dans les structures et d'adapter les contenus de l'enseignement à ses besoins nouveaux (création de sections professionnelle, industrielle, commerciale, etc.), ce dont allaient profiter les L2. Ainsi, en 1845, parmi les établissements scolaires situés dans les provinces flamandes subsidies par l'État, l'allemand était enseigné dans sept établissements et l'anglais dans six (sur neuf, y compris l'Athénée de Bruxelles) ${ }^{8}$. Dans un établissement exclusivement communal comme celui de Gand l'on enseignait deux L2. Les titulaires de ces cours étaient le plus souvent des natifs, appelés " maîtres de langues $»^{9}$. Ultérieurement, les professeurs diplômés se sont souvent gaussés de ces anciens maîtres sans diplôme, incapables, disaient-ils, de s'élever aux beautés littéraires d'un texte ou de le traduire correctement en langue maternelle. Selon Maréchal (op. cit. 77) « le savoir pédagogique leur faisait totalement défaut ». Mais d'après les travaux plus récents de Clavères (1989) sur les maitres d'anglais en France, ces jugements relèveraient plus d'une construction idéologique que de la réalité. Il semble en tout cas difficile de ne pas reconnaître à l'enseignement de ces maîtres une certaine efficacité qui correspondait à la finalité pratique généralement accordée aux cours de L2 par les contemporains (Baron 1849 : 55):

Une langue moderne ne s'apprend point comme un moyen de gymnastique intellectuelle [...]. Elle est son propre but. [...] ; il s'agit d'abord et surtout d'entendre et de parler, d'habituer l'oreille à saisir et la langue à reproduire des sons [...] en les prenant par groupes, en quelque sorte, et sans trop s'inquiéter de l'analyse rigoureuse. [...] ; beaucoup de mots, beaucoup d'idiotismes, les dialogues, les conversations familières, les journaux, le théâtre,... Savoir l'anglais et l'allemand c'est pouvoir converser intelligemment et écrire correctement en allemand et en anglais, n'eût-on lu une ligne de Goethe ou de Shakespeare, n'eût-on jamais ouvert Schiller ni Milton ${ }^{10}$.

\section{L'intervention de l'État: survol du processus d'institutionnalisation des L2}

\subsection{De 1850 à $1870:$ une relative stabilité}

9 L'introduction des L2 dans les programmes officiels s'accompagnera d'une argumentation valorisant systématiquement les vertus formatrices de cet enseignement sur base des qualités littéraires des L2. Le rapport de Trasenster (1849) ${ }^{11}$ qui a servi à l'élaboration du programme de la section professionnelle des établissements d'enseignement moyen, en est un des premiers témoignages ${ }^{12}$. Cette " mise à égalité » des L2 avec les langues anciennes se fera en parallèle avec une mise à niveau des enseignants de L2 aussi bien sur le plan de la formation (institution d'un diplôme de capacité en 1863, rattachement d'une Section pour professeurs de tangues vivantes à l'École normale des Humanités de Liège en 1874, création d'une Section de 
Philologie germanique dans les universités d'État en 1890 , etc.) que sur celui du statut (rémunération progressivement alignée sur celle des autres enseignants).

Les programmes élaborés en application de la loi de 1850 étaient, selon l'habitude de l'époque, assez brefs ${ }^{13}$. Ils plaçaient en première ligne $1^{\prime}$ « instruction littéraire », c'està-dire la capacité à lire, comprendre, imiter, voire produire des discours. Cette instruction se faisait au moyen des langues anciennes dans la section des humanités (auxquelles s'ajoutait l'étude de trois ou quatre langues modernes) et uniquement au moyen des langues modernes dans la section professionnelle (français, flamand, allemand et anglais) ; au moyen du français et du flamand dans les écoles moyennes ${ }^{14}$. Les programmes donnaient aussi, par classe, quelques indications sur les contenus et sur les techniques d'enseignement (lecture à haute voix, exercice de récitation, traduction de vive voix, thème d'imitation, etc.). Durant une vingtaine d'années ces textes officiels ne vont guère être modifiés. Peut-être faut-il voir dans cette stabilité le signe d'un certain consensus sur la place des L2 dans le cursus scolaire et sur les méthodes à suivre, à moins qu'il ne s'agisse d'un relatif désintérêt...

\subsection{De 1870 à 1884 : accélération du processus 'intégration des L2}

11 À partir des années soixante-dix, les L2 retiendront davantage l'attention des ministres ayant en charge l'enseignement et du Conseil de perfectionnement ${ }^{15}$. Une Commission des langues vivantes instituée en 1872 proposera une série de mesures. Certaines seront appliquées immédiatement (création d'un enseignement normal pour la formation de professeurs de langues modernes en 1874), d'autres verront leur application différée dans le temps (la rédaction de grammaires uniformes pour toutes les langues enseignées dont le concours se déroulera en... 1883-1886) ; certaines enfin seront reportées sine die (la concentration de l'enseignement des trois langues germaniques dans les mains d'un seul professeur). De manière générale, le nombre d'heures accordé à l'enseignement des L2 augmente.

Comme un peu partout en Europe, au niveau des programmes, la « cassure » s'opère au début des années 80 . Elle est l'œuvre du premier ministre de l'Instruction publique en Belgique, le libéral P. Van Humbeeck ${ }^{16}$. Sur le plan de l'enseignement linguistique, sa principale innovation est la création d'un socle commun de deux ans pour tous les établissements d'enseignement moyen dans lequel seules les langues modernes (français, flamand allemand) sont enseignées ${ }^{17}$. Cette loi comprenait encore d'autres dispositions importantes pour l'enseignement des L2 : il devient obligatoire dans les écoles moyennes ; dans les provinces flamandes il doit se faire via le flamand ${ }^{18}$. Le gouvernement s'engageait aussi à nommer dans les provinces flamandes des professeurs capables d'enseigner l'allemand et l'anglais par le flamand (ce qui sera formellement prescrit - mais pas toujours appliqué -par la loi linguistique de 1883). Il est à noter cependant que la loi de 1881, même si elle parle de « langues nationales » est loin d'instaurer une égalité entre le français et le flamand : le français reste première langue sur tout le territoire tandis que le flamand n'est que troisième langue en Wallonie où l'allemand lui est souvent préféré ${ }^{19}$. Cette différence est encore confirmée par les programmes de 1906 qui traitent d'un côté les langues anciennes et le français (programme identique pour tout le pays) et d'un autre les langues germaniques (programme variable selon le régime linguistique). Enfin, sur le plan pédagogique, ce qui frappe le plus à la lecture des programmes de 1880, c'est la grande convergence 
entre les cours de LI et de L2 pour ce qui est des techniques d'enseignement. Seuls les exercices de thèmes et de versions sont encore spécifiques à L2. Dans l'enseignement primaire et normal, la méthode recommandée pour l'enseignement des L2 est la méthode maternelle, c'est-à-dire celle que la mère emploie naturellement avec son enfant.

\subsection{De 1885 à 1914 : achèvement d'une étape ?} catholiques homogènes qui se succéderont de 1884 à 1914, le "socle commun » sera supprimé. La section professionnelle est transformée en " humanités modernes " (arrêté du 31/8/1887). Les langues modernes se voient ainsi confirmées dans leur valeur pédagogique puisque désormais elles peuvent aussi conduire aux « humanités ». D'autres indices confirment le processus d'intégration des L2 dans le cursus scolaire : fondation d'une Union des professeurs de langues modernes (1885), publication d'une revue L'enseignement des langues modernes (1887-1898) ${ }^{20}$ création d'une inspection spécifique (1895), premières interventions de l'État dans le domaine méthodologique (circulaires de 1897, de 1899,...), apparition d'une nouvelle Association des professeurs de langues vivantes (1906), organisation de congrès (Gand 1906 ; Liège 1909 ; Bruxelles 1911), etc. occidentale par ce qu'on a appelé (à tort, à notre avis) une « révolution méthodologique » (Puren 1988), c'est-à-dire l'essor de la méthode dite directe. Cette méthode entend mettre l'élève en contact direct avec les mots et les choses, sans le détour de la traduction, du métalangage, du signe écrit, etc. Elle a d'évidentes parentés avec la méthode maternelle ${ }^{21}$. Or, la figure de la mère, modèle de l'enseignement de la langue... maternelle, est une constante de tout le XLXe siècle. Ce qui se passe dans les années 1880-1900 est sans doute plus d'ordre institutionnel que méthodologique.

En Belgique, l'expression méthode directe ${ }^{22}$ apparaît pour la première fois en 1884 (même date qu'en Allemagne, cf. Reinfried $1999: 210$ ) dans la préface d'un manuel destiné aux instituteurs et rédigé par Sluys et Kesler, deux instituteurs à l'École modèle (1875-1880), fondée par la Ligue de l'enseignement. Au début des années 1890, l'on assiste à un véritable tir groupé de l'École normale de l'État à Lierre en faveur de la méthode directe : son directeur, H. Temmerman publie une brochure (1893) d'une trentaine de pages où il fait son éloge et en explique les fondements théoriques ; la même année, un certain Leflot, directeur de l'École d'application annexée à l'École normale, publie un manuel, le premier en Belgique à contenir dans son titre l'expression méthode directe ${ }^{23}$. Nous avons montré ailleurs (Berré, 2001) comment cette méthode permettait de concilier l'idée d'une Belgique unitaire tout en maintenant l'homogénéité linguistique de chaque communauté. À partir de 1897, diverses circulaires ministérielles enjoindront les enseignants à pratiquer la méthode directe, sans toutefois interdire le recours à la traduction " à titre exceptionnel ", ni supprimer l'enseignement systématique de la grammaire, " le seul en état de donner une connaissance complète et durable de la grammaire » ( $\hat{1})^{24}$. En 1895, le premier inspecteur de l'enseignement des langues germaniques sera nommé, il s'agit de J.-C. Kleyntjens auquel succédera J. Duqué (1907-). Enfin, signalons qu'à l'exception de quelques publications éphémères et de peu de diffusion, il faudra attendre 1935 pour voir réapparaître une revue spécifiquement destinée à défendre les intérêts des professeurs de langues vivantes. Signe des temps, 
elle sera bilingue (Revue des langues vivantes... Tijdschrift voor levende talen...) alors que la revue de l'Union des professeurs de langues modernes, rédigée en français, se contentait d'insérer des articles en différentes langues (flamand, anglais, allemand, etc.). Le rédacteur en chef de la Revue des langues vivantes est F. Closset, germaniste de l'Université de Liège, auteur en 1950 d'une Didactique des langues étrangères qui fut traduite en de nombreuses langues et où il défend l'idée d'une méthode éclectique.

\section{Quelques réflexions à propos du « progrès " dans l'enseignement des L2}

16 Après un survol aussi rapide il ne saurait être question de vouloir établir un bilan de l'intervention de l'État dans l'enseignement des L2, que ce soit en termes quantitatif ou qualitatif. $L$ 'on se contentera de trois remarques.

17 - À la veille de la première guerre mondiale, les L2 ont toutes les apparences d'une discipline scolaire comme les autres : elles disposent de professeurs spécialisés, de programmes spécifiques, d'un service d'inspection, d'une association de professeurs, etc. Et cependant, cinquante après l'arrivée des premiers professeurs diplômés, l'heure est plutôt à un certain désenchantement quant à la possibilité de mettre sur pied un enseignement des L2 efficace dans un cadre scolaire. "Ceux qui prétendaient apprendre à leurs élèves, l'emploi relativement courant d'une langue, faisaient naufrage et n'arrivaient à rien du tout ", constate l'abbé Colle au Congrès de BonneEspérance (1913: 523).

18 - Ce scepticisme pose, en second lieu, la question de la possibilité d'enseigner des L2 dans un cadre scolaire et celle du progrès dans l'enseignement. Un professeur diplômé enseignait-il mieux ou moins bien l'allemand en 1910 que ne le faisait un certain Kortländer à Tirlemont en 1845 ? Se poser la question (sans même y répondre), c'est déjà reconnaître que la notion de progrès, si souvent convoquée dans les débats sur l'enseignement des L2, n'est une notion ni évidente, ni " neutre " (inscrire l'enseignement dans la voie du progrès, c'est l'insérer dans un certain type de valeurs...). Mieux connaître le passé pour entre autres mieux maîtriser conceptuellement le présent, c'est un des enjeux (illusoire ?) de l'histoire de la didactique des langues.

19 - Enfin, si la législation de l'enseignement moyen prend en compte dès 1850 la diversité linguistique du royaume, le français reste dans les textes officiels la première langue de l'enseignement moyen dans les athénées et collèges flamands. La néerlandisation du niveau moyen ne se fera que très progressivement surtout dans les collèges catholiques où le flamand a certes une place mais de second rang. L'allemand et l'anglais ne sont en Flandre que troisième et quatrième langues. La langue allemande semble avoir eu la préférence jusqu'en 1914 entre autres parce que l'anglais, ayant la réputation de ne pas avoir de grammaire, la langue de Goethe était considéré comme plus formative pour l'esprit. À cela s'ajoutent d'autres facteurs comme le prestige de la science allemande, le fait que dans certaines régions du royaume la langue allemande était langue maternelle et que l'École normale des Humanités qui formait les professeurs pour l'instruction littéraire se trouvait à Liège, ville qui a historiquement des liens privilégiés avec la langue et la culture allemande. L'absence des langues romanes autres que le français est aussi une constante des programmes officiels au XIXe siècle. 


\section{Conclusion}

Est-il besoin de rappeler, presque sous forme de boutade, la légendaire facilité des Flamands à apprendre les langues étrangères ? Jacotot qui en avait entendu parler l'attribuait, non à une disposition naturelle, mais à la structure particulière de la langue flamande où ce qu'il appelle les « syllabes significatives » sont particulièrement visibles (1824 : 23-25). C'est cette connaissance des « radicaux » et la capacité à les repérer dans une langue nouvelle qui leur rendaient, selon Jacotot, l'étude des langues étrangères plus facile, surtout s'ils connaissaient le latin qui leur permettaient de « deviner [...] les autres idiomes (sic) du Midi qui en dérivent " (ibid.). Plus souvent, l'on attribue cette (prétendue ?) facilité à la diffusion relativement restreinte de la langue néerlandaise. L'on peut en effet admettre qu'il est plus motivant d'apprendre une langue davantage répandue que la sienne, surtout quand celle-ci est proche géographiquement et jouit d'un certain prestige (ce qui est le cas -dans des proportions qui ont varié au cours de l'histoire - du français, de l'anglais ou de l'allemand...). En additionnant l'argument linguistique de Jacotot et l'explication sociolinguistique, il n'est pas interdit de penser que du point de vue de l'enseignement des langues étrangères, l'étudiant flamand qui, vers 1860 , connaissait " par principes " sa langue maternelle, avait la maîtrise du français et une certaine connaissance du latin se trouvait dans une situation assez favorable pour apprendre d'autres L2.

\section{BIBLIOGRAPHIE}

ANONYME. 1829. Institut dirigé par E Gaggia, Glacis du faubourg de Namur, $n^{\circ} 92 b i s, a ̀$ Bruxelles. Plan de l'institution. Bruxelles.

ANONYME. 1913. Compte rendu des travaux du 'Congrès national de l'enseignement moyen libre', tenu à Bonne-Espérance les 11, 12, 13 septembre 1911. pd. en néerlandais]. Roulers : De Meester.

BAGUET, François. 1843-1863. De l'enseignement moyen. Bruxelles : Closson. BERRÉ, Michel. 2001. « Le français à l'école primaire en Flandre vers 1880-1890:

identités nationales et techniques d'enseignement ». KOK-ESCALLE - MELKA (éds). 235-252 .

Biographie nationale. 1866-1977. Bruxelles (Académie royale des Sciences, des Lettres et des beauxArts de Belgique).

BOERMA, T. 1885. De Fransche taal, volgens de rechtstreeksche méthode [= méthode directe]. Volledige cursus te voorbereiding van middelbaar en gymnasiaal on-derwijs. le cursus : directe aanschouwing. Groningue : J.-B. Wolters.

Documents pour l'histoire du français langue étrangère ou seconde, 26 | 2001 
BUISSON, Ferdinand (dir.). 1911. Nouveau dictionnaire de pédagogie et d'instruction primaire. Paris : Librairie Hachette et Cie.

CHERVEL, André. 1998. La culture scolaire. Une approche historique. Paris : Belin.

CLAVÈRES, Marie-Hélène. 1989. « Nous, les maîtres de langues ». Les langues modernes 1. 7-14 ; 2.11-16; $3-4.10-16 ; 5.7-14 ; 6.7-11$.

CLOSSET, François. 1950. Didactique des langues vivantes. Bruxelles : Didier.

COLLE (abbé). 1913. « Le séjour à l'étranger et la préparation à l'enseignement de la prononciation ». In : ANONYME 1.1. 622-627.

DE PAEPE, Jean-Lue - RAINDORF-GÉRARD, Christiane (avec la coll. de GILLARD, Patrick et BRION, René). 1996. Le Parlement belge 1831-1894. Bruxelles : Duculot (Académie royale de Belgique. Commission de la biographie nationale).

État de l'instruction moyenne en Belgique 1842-1848. 1849. Bruxelles : Devroye et Cie.

GARCIA, Jean-François. 1997. Jacotot. Paris : Presses universitaires de France.

GENONCEAUX, Louis. 1874. L'enseignement des langues modernes par Paul Donau. Bruges : De Moor.

GERMAIN, Claude. 1993. Évolution de l'enseignement des tangues : 5000 ans d'histoire (Coll. Didactique des langues étrangères). Paris : Clé International.

GESELSCHAP, Théodore. 1853. Essai sur l'étude des langues modernes, d'après les principes de W. V.

Humboldt. Gand : E. Vanderhaeghen.

GEVERS, Lieve. 1980. Kerk, onderwijs en Vlaamse beweging. Documenten uit kerke-lijke archieven over taalregime en vlaamsgezindheid in het Katholiek Middelba-ar Onderwijs (Interuniversitaire Centrum voor Hedendaagse Geschiedenis 89). Leuven : Nauwelaerts.

GROOTAERS, Dominique (dir.). 1998. Histoire de l'enseignement en Belgique. Bruxelles : Éditions du Centre de recherche et d'information socio-politiques.

GROOTAERS, Dominique (dir.). 1998. « Tensions et ruptures dans le projet éducatif et culturel des humanités (1830-1950) ». In : Grootaers 255-302.

ЈАСОТОт, Joseph. 1823. Enseignement universel. Langue maternelle. Louvain : De Pauw.

ЈАСОТОТ, Joseph. 1824. Enseignement universel. Langue étrangère. Louvain : De Pauw.

KOK-ESCALLE, Marie-Christine - MELKA, Franchie (éds). Changements politiques et statut des langues. Histoire et epistemologie 1780-1945. Amsterdam/Atlanta: Rodopi..

(De) Lagere school. Weekblad over onderwijs en opvoeding (1883-1887).

LAURENT, François. 1881. Enquête scolaire. Mémoire sur l'expertise des écoles normales publiques et privées. Bruxelles : Bruylants-Christophe \& Cie.

LEFLOT, Prosper. 1893. Cours pratique de français basé sur la méthode directe à l'usage des écoles néerlandaises, allemandes et anglaises (5 p.). Anvers : DeVreese.

MARÉCHAL, Raymond. 1972. Histoire de l'enseignement et de la méthodologie des langues vivantes en Belgique, des origines au début du XXe siècle. Enseignement secondaire officiel. Paris - Bruxelles Montréal : Didier.

Organisation de l'enseignement des langues germaniques dans les athénées royaux. Programmes et méthode. 1905. (Ministère de l'Intérieur et de l'Instruction publique. Administration de l'enseignement moyen. Enseignement moyen du degré supérieur). Bruxelles : Goemaere. 
Plan provisionnel d'études. 1777 (tiré à part).

PUREN, Christian. 1988. Histoire des méthodologies de l'enseignement des langues (coll. Didactique des langues étrangères). Paris : Clé International.

Rapport triennal sur l'état de l'enseignement moyen en Belgique (16 vol.). 1856-1901. Bruxelles [différents imprimeurs].

REINFRIED, Marcus. 1999. «Le mouvement réformiste et la méthode directe en Allemagne : développement, fondement théorique et variations méthodologiques ». In : SALEMA - KAHN - TEKEIRA (éds). 204-226.

SALEMA Maria-José - KAHN Gisèle - TEKEIRA Luis-Filipe (éds). 1999. L'enseignement de ta langue et de la littérature françaises dans ta seconde moitié du xixe siècle. Actes du colloque de la S.I.H.F.L.E.S. (Société internationale pour l'histoire du français langue étrangère et seconde) organisé à Sintra - Portugal du 1 au 3 octobre 1998. Documents pour l'histoire du français langue étrangère et seconde 23. Paris. SLUYS, Alexis. 1912. Geschiedenis van het onderwijs in de drie graden in Begië tij-dens de Fransche overheersching en onder de regeering van Willem I (Koninklijke Vlaamsche Académie voor taal- en letterkunde, Bekroonde werken 6). Gent : Siffer.

SLUYS, Alexis - KESLER, Johan. 1884. Les deux langues nationales. Méthode pratique pour enseigner le français aux Flamands et le néerlandais aux Wallons. Exercices d'élocution. [Id. en néerlandais]. Gand Gent : Annoot-Braeckman -Hoste.

TARUER, H. (éd.) 1840-. Almanack royal de Belgique. Bruxelles : Librairie polytechnique, A. Decq (années 1840,1841 et 1845).

TEMMERMAN, Hippoliet. 1893. Aanvankelijke Studie der vreemde levende talen. Gent : Hoste.

TRASENSTER, Jean-Louis. 1849. «Rapport adressé par M. Trasenster, inspecteur des études à l'école des arts et manufactures et des mines de Liège, à M. le Ministre de l'Intérieur, sur la situation de l'enseignement industriel dans les collèges (sic) et autres écoles moyennes, en 1848 ». In : État de l'instruction moyenne. 435-467.

TYSSENS, Jeffrey. 1998. «L'enseignement moyen jusqu'au Pacte scolaire : structuration, expansion, conflits ». In : GROOTAERS 221-254.

VAN DER MEULEN, R. 1892. Brinkman's Catalogus der boeken, plaat- en kaartwer-ken, die sederi 1882 tot en met 1891 in Nederland zijn uitgegeven of herdrukt. Amsterdam : C. L. Brinkman [facsimile-uitgave ; Leiden : A.-W. Sijhoff, 1966].

\section{NOTES}

1. L2 signifie donc ici les autres langues que la langue maternelle et que le français, c'est-à-dire, sauf indication contraire, l'allemand et/ou l'anglais. Ll désigne la langue maternelle de l'élève, faisant abstraction des différences entre dialectes, néerlandais de Belgique et néerlandais des Pays-Bas.

2. Laurent (1881:68).

3. Sur le statut du français en Flandre, quelques remarques et indications bibliographiques dans Berré (2001).

4. Rappelons que le français figurait au programme des établissements d'enseignement moyen.

5. La bourgeoisie francophile de Bruxelles avait de multiples raisons de se méfier de Jacotot : l'oreille bienveillante que lui accordait le roi des Pays-Bas (qui espérait tirer profit de sa méthode pour faciliter la diffusion du néerlandais dans la partie méridionale du pays) agaçait ceux qui 
s'opposaient à la politique de néerlandisation de Guillaume; les opinions de Jacotot à propos des langues (tout n'y est que convention, l'ordre des mots est arbitraire) et des intelligences (elles sont toutes égales) ne pouvaient qu'inquiéter, par leurs implications sociales, les « classes possédantes ». Pour une récente synthèse sur Jacotot, cf. Garcia (1997).

6. Cf. Anonyme (1829).

7. En 1846-1847, le clergé y contrôlait 83\% des écoles secondaires (Gevers 1980).

8. D'après l'Almanack royal (1845), cf. Tarlier (éd.).

9. Cf. les noms de quelques professeurs d'allemand : Lebermuth à Bruxelles, Kortländer à Tirlemont, Kronenberger à Bruges, Schmeisser à Louvain, etc. (Almanack royal $1841: 451$ et 1845 : 464-465 ; cf. Tarlier).

10. Sur Baron, professeur à l'Université de Bruxelles, cf. BN t. XXLX (204-212).

11. Sur Trasenster, professeur à l'Université de Liège, cf. BN t. XXV (545-547).

12. «[...] deux langues modernes [l'auteur a ici en vue LI et une L2] bien sues [sont] un exercice littéraire beaucoup plus profitable que deux langues anciennes apprises avec cette négligence qui se remarque presque partout dans nos collèges (sic). J'ai la conviction aussi qu'un homme nourri de la lecture de Shakespeare (sic), de Schiller, de Goethe, aura de la nature humaine, de sa dignité, de sa destinée, des idées beaucoup plus vraies et profondes que celui qui ne connaît qu'Homère et Virgile, Anacréon et Horace » (Trasenster 1849 : 438).

13. La loi de 1850 « dessine une architecture d'ensemble pour l'enseignement moyen officiel, comprenant deux formes d'humanités complètes (toutes les deux données dans les athénées, chacune poursuivant une finalité sociale spécifique et s'adressant à un public distinct, ainsi qu'une forme d'enseignement moyen de type court (donnée dans les écoles moyennes), destinées à un public d'origine sociale plus modeste " (Tyssens $1998: 271)$. Pour rédiger les paragraphes repris sous 2.1., 2. 2. et 2.3., nous avons consulté les RTEM(1856-1901).

14. Le « bilinguisme » de l'enseignement moyen de type court ne vaut que pour le nord du pays. Dans les provinces wallonnes, le flamand n'est que matière facultative et ne sera guère enseigné. 15. C'est également de cette époque (1874) que date le premier ouvrage consacré spécifiquement à l'enseignement des langues vivantes, écrit par un auteur belge, L Genonceaux, professeur à l'École normale de l'État à Bruges. L'auteur se sert d'un pseudonyme (P. Donau). L'ouvrage semble malheureusement perdu. Maréchal (qui croit avoir affaire à un professeur Donau !) en cite des extraits (op. cit. 177-198).

16. Indications bio- bibliographiques dans De Paepe et al. 1996

17. Cette idée n'était pas nouvelle. Elle figurait déjà dans les cartons du département de l'instruction publique en 1831-1832 et fut reprise entre autres par le professeur d'université Baguet au milieu du XIXe siècle

18. À vrai dire, les enseignants n'ont pas attendu la fin du XIXe siècle pour " découvrir " des analogies entre les trois langues germaniques. Dès 1830 (et sans doute avant), l'on trouve des ouvrages pédagogiques signalant les ressemblances au niveau morphologique et syntaxique entre le flamand l'allemand et l'anglais mais ces ouvrages étaient le plus souvent rédigés en français. Ce qui est enjeu dans les réformes successives de 1880 et 1883, c'est moins la pédagogie des L2 qu'un rapport de force (en train de basculer) entre le flamand et le français dans l'enseignement moyen en Flandre.

19. Les établissements situés dans les provinces wallonnes avaient cependant la possibilité d'opter pour le régime linguistique flamand, à savoir : le français comme $\mathrm{Ll}$, le flamand, comme seconde langue et l'allemand comme troisième langue...

20. Cette revue animée par son directeur Th. Hegener combattra certains principes de la méthode directe. On peut supposer que sa disparition (et celle de l'association qui la soutenait) est liée à cette lutte qui dans les années 1890 pouvait passer pour un combat d'arrière-garde.

21. Cf. Germain (1993 : 131) : «La méthode directe repose sur le postulat de la similarité d'apprentissage d'une Ll en milieu naturel, et d'une L2 en contexte scolaire ». 
22. Les termes immédiat, direct se trouvent déjà sous la plume d'un auteur comme Geselschap (1853).

23. Aux Pays-Bas, ou trouve un tel ouvrage dès 1885 (cf. Boerma et pour les références Van der Meulen). Une annonce pour cet ouvrage se trouve dans la revue belge De Lagere school (1885-IR : 127).

24. Pour ces deux citations, cf. Organisation de l'enseignement des langues germaniques 1905 : 16-17.

\section{AUTEUR}

\section{MICHEL BERRÉ}

Vrije Universiteit Brussel 